\title{
Clinicopathological correlation of P63 in Urothelial Carcinoma: Immunohistochemical Study
}

\author{
Rupinderjit Kaur*, Mridu Manjari and Sanjay Piplani \\ Department of Pathology, Sri Guru Ram Das Institute of Medical sciences and Research
}

\begin{abstract}
Background: Urothelial cancer (UCa) poses a significant medical and public health concern in most parts of the world and is defined as a pan- urothelial disease in which entire urothelium of the renal pelvis to the urethra can undergo malignant transformation. Bladder tumors account for $90-95 \%$ of urothelialcarcinomas and are the most common urinary tract malignancy. It is three times less common in females.

Methods: The study was conducted on 40 cases of Urothelial Carcinoma received as biopsy specimens in atertiary care hospital. There were 20 cases each of low grade and high grade and after grading these were subjected to p63 expression.

Result: $78 \%$ of the patients werefrom 50-75year age group with Male to Female ratio being 4:1. Painless hematuria was most common complaint. The size varied from 0.5 to $7.2 \mathrm{~cm}$.

For P63, 38 (95\%) cases showed positive p63 expression. Of which 20 cases were low grade and 18 (90\%) were high grade papillary urothelial carcinomas. Of P63 positive cases 36 cases showed moderate to strong staining intensity whereas two cases showed mild staining intensity. The p63 expression decreased with grade and invasion.

Conclusion: P63 immunoexpression decreases with increasing grade of tumor as well as with invasion thus showing that its expression is related with good prognosis.
\end{abstract}

Keywords: p63, Urothelial Carcinoma, Immunohistochemistry, Grade Of Tumor

\section{Introduction}

Urothelial cancer is the seventh most common cancer worldwide and is a significant global health problem, with an estimated 260,000 new cases occurring each year in men. Bladder tumors account for $90-95 \%$ of urothelial carcinomas (UCa) and are the most common urinary tract malignancy. Bladder cancer should be considered an aggressive and potentially lethal disease. ${ }^{[1]}$

$\mathrm{UCa}$ is more common in developed countries and is fourth and ninth most common cancer in men and women respectively in western world. Urinary Bladder cancer (UBC) is more common among men than in women, in industrialized than in developing nations and in urban areas than in rural dwellers. ${ }^{[2]}$

The incidence rate increases with age in log linear fashion. In India, the mean age of presentation is $60.2 \pm 4.0$ years with the male to female ratio being $8.6: 1 .{ }^{[3]}$

Bladder cancer is the fourth most common cancer in men in the United States, after prostate, lung, and colorectal cancer. It is three times less common in women than in men. 81,190 new patients were diagnosed and it lead to 17,240 deaths in 2018 in US. ${ }^{[4]}$

There are several known potential risk factors of bladder cancer. Cigarette smoking, occupational exposure, Chronic infection, irritation, calculi (staghorn) predispose to UBC. ${ }^{[5]}$ Arsenic exposure in drinking water is a factor in the development of bladder cancer along with workers in chemical, petrochemical, aniline dye and plastic industry as well as those exposed to coal, tar, asphalt are also at increased risk . Chronic cystitis caused by Schistosoma haematobium is a known cause and the resultant bladder tumors are usually squamous cells carcinomas. ${ }^{[6]}$

A complete urine examination, urine culture, cytology, cystoscopy, various radiological investigations like Renal ultrasonography, Intravenous urography (IVU), and other radiological imaging modalities are used to diagnose UBC early. ${ }^{[7]}$

Transitional cell carcinoma(TCC)now classified as papillary urothelial carcinoma is most frequent type followed by Squamous cell carcinoma and Adenocarcinoma. ${ }^{[8]}$

In recent years value of Immunohistochemistry (IHC) in Urothelial carcinoma has raised as an important tool not only for diagnosis of the type but also in predicting prognosis. The p63 a nuclear protein encoded by gene on chromosome $3 q 27-29$, is a member of the p53 gene family. P63 is mainly expressed in the basal cell compartment of the stratified epithelium, including the urothelium.In urothelial carcinoma, decreased p63 expression is associated with 
more advanced disease and poorer prognosis and decreased expression of p63 is associated with higher grade and TNM stage. ${ }^{[9]}$

\section{Materials and Methods}

The study was conducted on 40 cases of Urothelial Carcinoma received as biopsy specimens in a tertiary care hospital. The tissue was formalin fixed and paraffin embedded and was then stained for haematoxylin and eosin for Histopathological staging and grading. Grading was done according to World Health Organization classification of tumors (2016) and staging according to American Joint Committee on Cancer (2018). ${ }^{[2]}$ This was followed by Immunohistochemical staining with p63 using Primary antibody - Mouse Monoclonal Antibody (Biomedical Care).

Positive and negative controls were run with every batch of the IHC. P63 is mainly a nuclear stain .p63 staining was studied and results were evaluated semi quantitatively using Ivan D et al scoring criteria. ${ }^{[10]}$ Brown nuclei were taken as positive.

\begin{tabular}{|c|c|c|c|}
\hline $\begin{array}{c}\text { Percentage } \\
\text { positivity }\end{array}$ & Score & $\begin{array}{c}\text { Staining } \\
\text { intensity }\end{array}$ & Score \\
\hline$<5 \%$ & 0 & Nil & 0 \\
\hline $5-25 \%$ & 1 & Mild & 1 \\
\hline $26-75 \%$ & 2 & Moderate & 2 \\
\hline$>75 \%$ & 3 & $\begin{array}{c}\text { Severe/ } \\
\text { Strong }\end{array}$ & 3 \\
\hline
\end{tabular}

A final immunoscore was calculated by adding scores of percentage positivity of cells and intensity and categorized as weak (1-2), moderate (2-4) and strong (5-6).

\section{Result}

Majority of the patients in this study were from 50 -75 year age group constituting $78 \%$ of cases. The youngest patient was 40 years of age and eldest was 90 years old. Mean age was 62.3 years. Male to female ratio was $4: 1$. Majority of patients (18 cases, $45 \%$ ) presented with painless hematuria as chief complaint followed by painless hematuria with burning micturition. Size of the tumor (radiologically) varied from 0.5 to $7.2 \mathrm{~cm}$.

Of the 40 cases taken there were equal number of i.e. 20 cases each of Low grade and High grade Papillary Urothelial Carcinoma. Among Low grade 19 cases were of Non-Invasive type and 1 case was Invasive whereas among High grade 18 cases were of Invasive type and 2 cases were Non-Invasive. ( $p$ value $=0.01$ ) (Figure.1). P63 positive immunoexpression was seen in 38/40 cases (95\%) of Urothelial carcinoma. All Low grade cases whether Invasive and Non-invasive were positive. In High grade tumors two Invasive cases were negative whereas in remaining 18, 2 Non-invasive and 16 invasive tumors were positive thus showing that as the grade as well as the invasion increased the p63 positivity decreased and was highly statistically significant with p value 0.00 . (Table 1 ).

In this study, of the total 28 biopsy specimens in which muscularis propria was present, 12 were low grade with rest being high grade. No muscularis propria invasion was seen in low grade but in high grade tumor six cases showed invasion in muscularis propria. $\mathrm{P}$ value- 0.284 which is statistically not significant. (Table 2)

One case from High grade Invasive type and another one from Low grade Invasive type showed Squamous differentiation in this present study and necrosis was present in $27.5 \%$ of cases and these were High grade cases.

P63 showed variation in its staining intensity expression. P63 was expressed in all cases of Low grade cases, as it was strongly expressed in 10/20 (50\%), moderately expressed in 9/20(45\%), weakly expressed in $1 / 20(5 \%)$ of cases. Amongst High grade two cases were negative for P63 and was strongly expressed in 9/20(45\%), moderately expressed in $8 / 20(40 \%)$, weakly expressed in $1 / 20(5 \%)$ with P value -0.997. (Figure 2) (Table 3)

Table 1: Correlation of P63 Positive Cases with Histological Grade of Tumor.

\begin{tabular}{|c|c|c|c|c|}
\hline Histological type & Lamina propria invasion & No. of cases & Positive cases & Percentage \\
\hline Low grade & Non invasive & 19 & 19 & $100 \%$ \\
\hline & Invasive & 1 & 1 & $100 \%$ \\
\hline High grade & Non invasive & 2 & 2 & $100 \%$ \\
\hline & Invasive & 18 & 16 & $89 \%$ \\
\hline
\end{tabular}

$p$ value $=0.000$ highly significant-chi square

$p$ value $<0.05$; significant,

$p$ value $<0.001$; highly significant,

$p$ value $>0.05$; insignificant 
Table 2: Showing Muscularis Propria Invasion.

\begin{tabular}{|c|c|c|c|}
\hline & $\begin{array}{c}\text { Muscularis propria Not } \\
\text { included }\end{array}$ & $\begin{array}{c}\text { Muscularis propria } \\
\text { invasion absent }\end{array}$ & $\begin{array}{c}\text { Muscularis propria } \\
\text { Invasion present }\end{array}$ \\
\hline Low grade Non Invasive type & 8 & 11 & - \\
\hline Low grade Invasive type & - & 1 & - \\
\hline High grade Non Invasive type & - & 2 & - \\
\hline High grade Invasive type & 4 & 8 & 6 \\
\hline Total cases & $\mathbf{1 2}$ & $\mathbf{2 2}$ & $\mathbf{6}$ \\
\hline
\end{tabular}

$p$ value $=0.284$ Not significant; chi-square

$p$ value $<0.05$; significant,

$p$ value $<0.001$; highly significant,

$p$ value $>0.05$; insignificant

Table 3: SHOWING STAINING INTENSITY

\begin{tabular}{|c|c|c|c|}
\hline Staining intensity & Low grade & High grade & Total \\
\hline Mild & 1 & 1 & 17 \\
\hline Moderate & 09 & 08 & 19 \\
\hline Strong & 10 & 09 & \\
\hline
\end{tabular}

$p$ value $=0.997$ Not Significant chi-square

$p$ value $<0.05$; significant,

$p$ value $<0.001$; highly significant,

$p$ value $>0.05$; insignificant

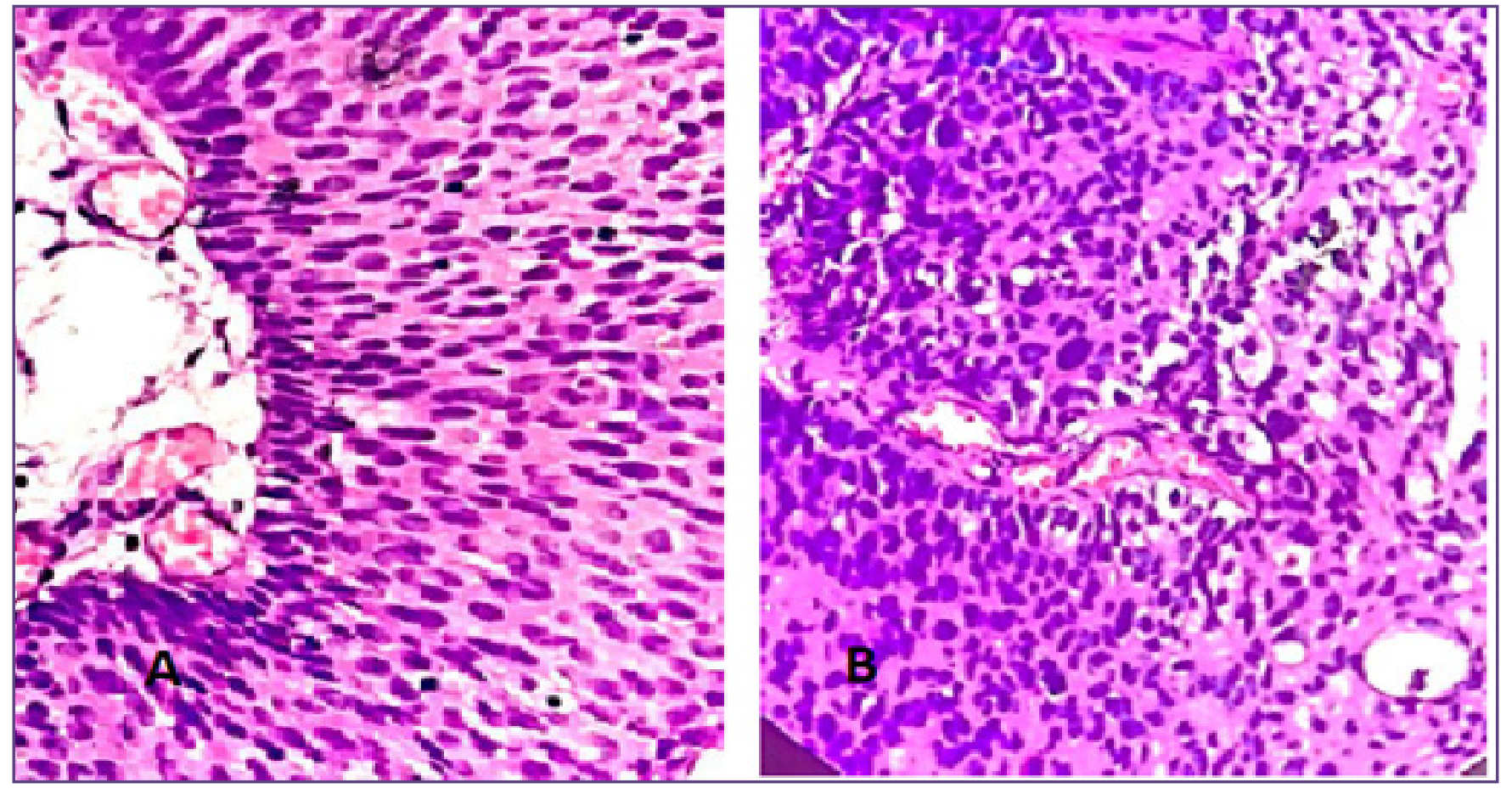

Fig. 1: (A) Microphotograph showing Low grade Papillary Urothelial Carcinoma (H\&E;100X) (B) High Grade Papillary Urothelial Carcinoma (H\&E; 100X). 


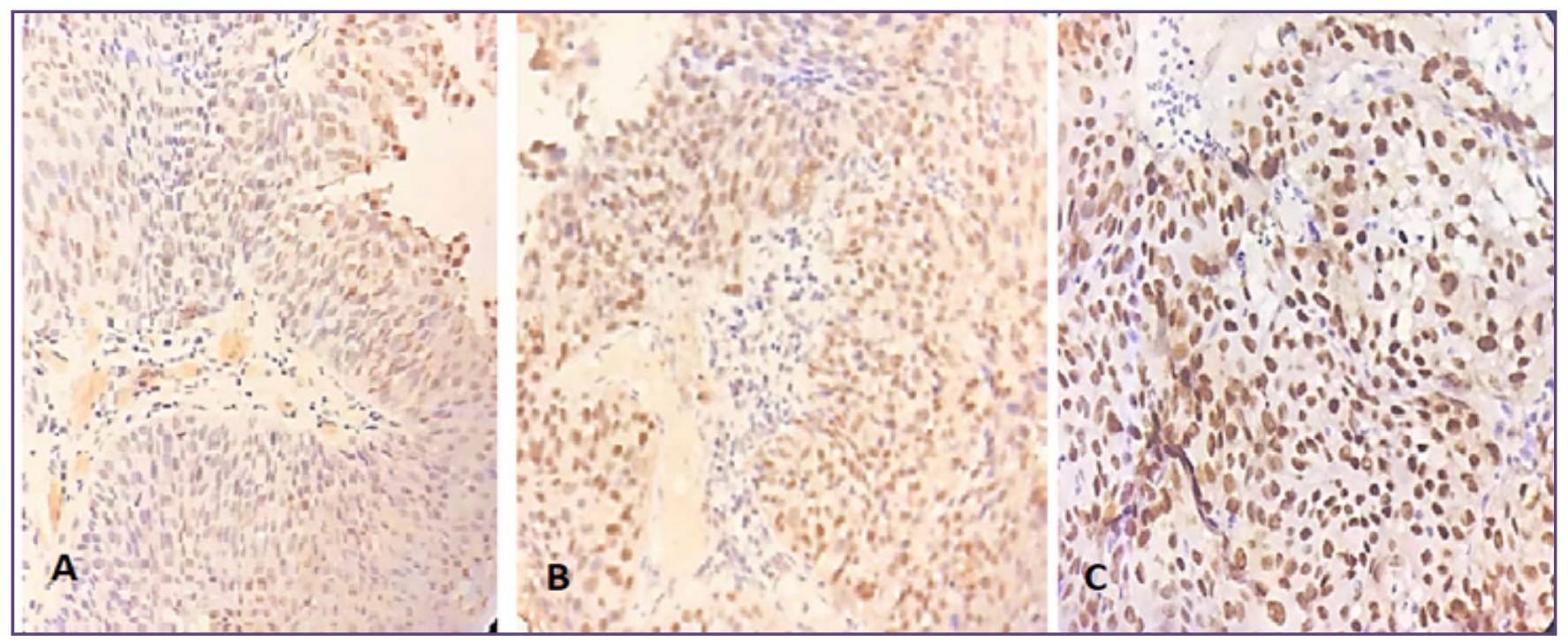

Fig. 2 :Microphotograph showing Positive p63 Staining (Nuclear), (A) Mild Staining Intensity (IHC;100X) (B) Moderate Staining intensity (IHC;100X), (C) Strong Staining Intensity (IHC;400X).

\section{Discussion}

Urothelial tumours comprise $90 \%$ of all urinary bladder neoplasms. TCC now classified as papillary urothelial carcinoma is most frequent type followed by Squamous cell carcinoma and adenocarcinoma. Based on clinical and histological observations $\mathrm{UCa}$ are divided into two types -Low grade Papillary Urothelial carcinoma (LGPUC) and High grade Papillary Urothelial carcinoma (HGPUC). High grade tumours are more of Invasive type than Low grade. The high grade papillary lesions primarily progress from flat dysplastic \& with carcinoma in situ changes to invasive lesions. ${ }^{[7]}$

The recent WHO classification of Urothelial tumours (2016) presents better definition criteria of pathological features useful in establishment of correct diagnosis and updates in the terminology and molecular characteristics of important subtypes of UBC. Also, in recent years improved pathological recognition and clinical understanding of how distinct variants of $\mathrm{UCa}$ can affect patient prognosis and this novel approach has impacted the process of therapy selection ${ }^{[1]}$

Various studies are being conducted on prognostic factors and tumor markers involved in initiation and progression of Urothelial carcinoma. P63 expression was evaluated to find out its expression in various grades of urothelial carcinoma.

In this study, majority of the patients were from 50-75 year age group and mean age was 62.3 years and presented with painless hematuria as most common complaint. Similar findings were observed by Elnashar TA et al. where patients were in age range of $20-80$ years. ${ }^{[12]}$

High grade papillary Urothelial carcinoma cases were more Invasive. Out of 20 High grade cases 18 were of Invasive type making up to $45 \%$ of total whereas amongst Low grade type only 1 case was Invasive. Our findings were similar with findings of Elnashar TA et al in their study where a total of 50 cases of UCa were taken, of which 17 cases were low-grade and 33 cases were high grade. Amongst LGPUC, 8 cases (47\%) were Invasive and rest were Non Invasive type. Whereas of HGPUC, 90\% cases were invasive. ${ }^{[12]}$

Of the total $38 / 40$ cases (95\%) were positive for p63 expression and two negative cases were High grade. All Low grade tumors whether Non Invasive or Invasive were positive for p63 whereas in High grade 18/20 showed positive p63 expression and two cases which were negative were Invasive type. It was observed that although p63 expression was seen in both low grade and high grade but percentage of $\mathrm{p} 63$ positive cells decreased with increasing histological grade of tumor. (Table 1) Our findings corroborate with findings of MarshalJ urist et al. where they found a statistically significant inverse association between the percentage of tumor cells positive for p63 and increasing pathological grade .LGPUC expressed p63 in $93 \%$ of tumor cells. However in the High grade, p63 positivity was $68 \%$. $(p=0.0002)$ which was statistically significant. ${ }^{[13]}$

Similarly in study by Ahmed RA et al. in 2019 , where a total of 66 cases of UCa were taken, of which 16 were Low 
grade and 50 were High grade, Amongst them $62 \%$ cases $(\mathrm{n}=10)$ of low grade , and $24 \%$ cases $(\mathrm{n}=12)$ of high grade showed positive $\mathrm{p} 63$ expression. $(\mathrm{p}=0.015) .^{[14]}$

Turan $\mathrm{T}$ et al. analyzed in their study conducted on 169 bladder cancer cases of which 106 showed invasion in Lamina propria. The cases with $\mathrm{pT} 1 \mathrm{~b}$ stage of superficial cancer (Invading to or near the muscularis mucosae (MM) showed high tumour progression rate of $76 \%$ as compared to those in pT1a stage (without invasion to or near to MM) that is $52 \%$. Hence they concluded that patients with superficial cancer invading to or near the MM should be followed up carefully for disease progression. ${ }^{[15]}$ Similarly in the present study of 28 cases in which Muscularis propria was present , 12 cases were low grade while the rest being high grade cases. Of these six cases showed Muscularis propria Invasion and all these were High grade and none was of low grade. (Table 2)

P63 Staining intensity slightly decreased with increase in grade of tumor .Our results are consistent with the findings of A.Stepan et al. in their study that p63-expression and Intensity of staining decrease with the depth of invasion of tumor, and is minimum in muscle invasive bladder carcinomas. ${ }^{[10]}$ (Table 3). P63 is expressed at high levels in normal Human Urothelium and neoplastic change is associated with diminished $\mathrm{p} 63$ expression pattern. Altered p63 expression in Urothelial carcinoma may occur with progressive loss of urothelial differentiation as tumors advance in both stage and grade

\section{Conclusion}

Thus it is concluded that as the p63 expression decreases with increasing grade of tumor and Muscularis Propria invasion, so all the cases of low grade or high grade papillary urothelial carcinoma, non-invasive or invasive if subjected to p63 expression and if expressed p63 can be used as a diagnostic and a prognostic factor for high grade and invasive cases of urothelial carcinoma, and timely institution of concurrent radiation therapy ,chemotherapy or radical cystectomy can improve the metastasis free survival of the patient.

\section{Funding}

Nil

\section{Competing Interests}

There are no conflicts of interests.

\section{References}

1. Roupret M, Babjuk M, Comperat E, Zigeuner R, Sylvester RJ, Burger M . European Association of Urology Guidelines on Upper Urinary Tract Urothelial Cell Carcinoma: 2015 Update. Eur Urol. 2015 Nov;68(5):868-79.

2. Grignon DJ, Lloreta J, Al Ahmadie H, Lopez-Beltran A, Amin MB, McKenney J, et al. Urothelial tumors: Infiltrating Urothelial carcinoma. In: Holger Moch, Peter A, Humphrey, Thomas M, Ulbright, Victoe ER, editors. (2016) World Health Organization Classification of Tumors. Pathology and Genetics of Tumors of the Urinary System and Male Genital Organs. 4th edition. Lyon (France): IARC Press; 2016. p.77-133.

3. Gupta P, Jain M, Kapoor R, Muruganandham K, Srivastava A, Mandhani A. Impact of age and gender on the clinicopathological characteristics of bladder cancer. Indian J Urol. 2009 Jun;25(2):207-10

4. Noone AM, Howlader N, Krapcho M, et al. SEER Cancer Statistics Review, 1975-2015, National Cancer Institute. Bethesda, MD.2017 Nov. [updated 2018 Apr cited on 2019 Oct]. Available online: https://seer.cancer.gov/ csr/1975_2015/

5. Pashos CL, Botteman MF, Laskin BL, Redaelli A. Bladder cancer: epidemiology, diagnosis and management. Cancer Pract. 2002 Nov-Dec; 10(6):311-22

6. Sharma S, Ksheersagar P, Sharma P. Diagnosis and treatment of bladder cancer. Am Fam Physician. 2009 Oct;80(7):717-23.

7. Heicappell R, Mattheis MV, Reinhardt M, Vosberg H, Gerharz CD, Ackermann R et al . Staging of pelvic lymph nodes in neoplasms of the bladder and prostate by positron emission tomography. Eur Urol . 1999 Dec; 36(6):582-7.

8. Cohen SM. Comparative pathology of proliferative lesions of the urinary bladder. Toxicol Pathol;30(6):663-71.

9. Orit KS, Mireia CM, Huai ST, Gladoun N, Domenech JD, Carbayo MS et al. Distinct Expression Profiles of p63 Variants during Urothelial Development and Bladder Cancer Progression. Am J Pathol. 2011 Nov;179(5):2674-5.

10. Stepan A, Margaretsu CA, Cristiana S, Raluca C. Ecaderin and p63 immunoexpression in dysplastic lesions and Urothelial carcinoma of bladder. Romanian journal of morphology and embryology. 2009 Jun;50(3):461-65.

11. Lopez-Beltran A, Venessa H, Montironi R, Cimadamore A, Raspollini MR, Cheng L. Variants and new entities of bladder cancer. Histopathology [Internet]. 2019 [cited on Jan 2020]; 74(1):77-96. Available from: https://onlinelibrary. wiley.com/doi/full/10.1111/his.13752.

12. Elnashar TA, Noha ED, Hassab EN, Ola MN, Altef G, Wahab EL, et al. Immunohistochemical expression of p63 in urothelial carcinoma. Smj. journals. 2017 Jan;21(1):237-46.

13. Urist MJ, di Como CJ, Lu ML, Charytonowicz E, Verbel D, Cordon-Cardo C, et al. Loss of p63 expression is associated with tumor progression in bladder cancer. American Journal of Pathology. 2002 Oct;161(4):1199-1206. 
14. Ahmed RA, Ibrahim Khalil EZ, Issam AA, et al. Prognostic role of epithelial mesenchymal transition markers ' $\mathrm{E}$ cadherin, b catenin, ZEB1, ZEB2, and p63' in bladder carcinoma. Wjon. 2019 December;10(6):199-217.
15. Turan T, Efilogu O, Gunaydin B, Ozkanli S, Nikerel E, Atis G. Comparative differences between $\mathrm{T} 1 \mathrm{a} / \mathrm{b}$ and $\mathrm{T} 1 \mathrm{e} / \mathrm{m}$ as substages in T1 urothelial carcinoma of bladder. Int Braz J Urol. 2018 Mar-Apr;44(2):267-72.

*Corresponding author:

Dr. Rupinderjit Kaur, Junior Resident ,Department of Pathology, Sri Guru Ram Das Institute of Medical sciences and Research, Vallah, Sri Amritsar. 143501 INDIA

Phone: +91 9501180747

Email: drrupibal@gmail.com,

Date of Submission : 27/11/2019

Financial or other Competing Interests: None.

Date of Acceptance : 13/04/2020

Date of Publication : 27/06/2020

Annals of Pathology and Laboratory Medicine, Vol. 7, Issue 6, June, 2020 